\title{
Nuevo registro de una serpiente del género Amastridium Cope (Serpente: Dipsadinae), para la selva natural chocoana de Colombia
}

\author{
New record of a serpent of the genus Amastridium Cope \\ (Serpente: Dipsadinae), from natural Chocoan forest of Colombia
}

\author{
Jonard David Echavarría-Rentería*, Jhon Tailor Rengifo-Mosquera*
}

\begin{abstract}
Resumen
Se registra una serpiente de la subfamilia Dipsadinae para la selva natural chocoana de Colombia. El nuevo registro es de Amastridium veliferum, que se considera como el segundo registro para el país y quien completa un vacío en la distribución de la especie, porque previamente fue reportada para la Cordillera Occidental en el municipio del Carmen de Atrato, departamento del Chocó, Colombia. Este segundo registro se hace con base en un ejemplar macho depositado en la Colección Científica de Referencia Zoológica del Chocó-Herpetología, de la Universidad Tecnológica del Chocó «Diego Luis Córdoba», colectado en el Cerro Tacarcuna, municipio de Unguía, departamento del Chocó, ubicado a los 8¹'30"N, $77^{\circ} 09^{\prime} 25^{\prime \prime} \mathrm{W}$, a una altura de $1876 \mathrm{~m}$. Este ejemplar presenta color café achocolatado, cabeza alargada, uno a dos escamas temporales, tubérculos supra-anales, 17 filas de escamas en todo el cuerpo y placa anal dividida, características típicas de la especie.
\end{abstract}

Palabras clave: Amastridium veliferum, Dipsadinae, Distribución, Selva chocoana.

\begin{abstract}
There is a snake subfamily Dipsadinae for natural forest Chocoana of Colombia. The new record is Amastridium veliferum, which is considered as the second record for the country and who completes a vacuum in the distribution of the species, as it was previously reported for the Cordillera Occidental in the municipality of Carmen de Atrato, department of Choco, Colombia. This second recording is made on the basis of an exemplary male deposited in the Colección Científica de Referencia Zoológica del Chocó-Herpetología, de la Universidad Tecnológica del Chocó «Diego Luis Córdoba», collected in the Cerro Tacarcuna, municipality of Unguia, department of Choco, located at the $8^{\circ} 1^{\prime} 30^{\prime \prime} \mathrm{N}, 77^{\circ} 09^{\prime} 25^{\prime \prime} \mathrm{W}$, at a height of $1876 \mathrm{~m}$. This exemplary presents chocolate coffee color, elongated head, one to two flakes temporary, tubersabove-annals, 17 . Rows of scales throughout the body and anal plate divided, typical features of the species.
\end{abstract}

Keywords: Amastridium veliferum, Dipsadinae, Distribution, Forest chocoana.

El género Amastridium Cope (1861) es poco conocido (Wilson 1988); sin embargo, está ampliamente distribuido en las vertientes pacífica y atlántica de Centroamérica hasta el corregimiento de Guaduas, municipio del Carmen de Atrato, departamento del Chocó, Colombia, entre 1610 y 1690 msnm (Rueda-Almonacid y Rueda-Martínez 2004). En Centroamérica habita desde el estado de Nuevo León (México) hasta la zona de Panamá, desde los 0 a 1520 msnm (Wilson y Johnson 2010). El género está incluido dentro de la subfamilia Dipsadinae (Pyron et al.
2013), que contiene dos especies A. sapperi (Werner 1903) y A. veliferum Cope (1891) (Uetz y Hallermann 2014). Estos dos taxones durante un tiempo se habían considerado como especies diferentes, separadas alopátricamente (Smith 1971, Savage 2002). Wilson y Meyer (1969) refutaron, que estos dos taxones eran la misma especie. La perspectiva de una especie de Amastridium duró varios años (Wilson y Meyer 1985, Wilson 1988) pero en 1971, Smith consideró que las poblaciones del norte presentaban una escama loreal y un número alto de ventrales (144-170) y que

* Universidad Tecnológica del Chocó, Facultad de Ciencias Básicas, Grupo de Investigación en Herpetología, Quibdó, Colombia. e-mail: jd07echavarria@hotmail.com

Fecha recepción: Junio 16, 2014 
las poblaciones del sur les faltaba la escama loreal, acompañado de un número relativamente bajo de ventrales (111-134) y concluyó que estos dos grupos se deberían reconocer como distintas subespecies. Este punto de vista ha sido seguido por otros autores (Savage 2002, Rueda-Almonacid y Rueda-Martínez 2004, Köhler 2008, Acevedo et al. 2010, Johnson et al. 2010, Stafford et al. 2010, Townsend y Wilson 2010, Wilson y Johnson 2010, McCranie 2011, Wilson et al. 2013, Lara-Tufiño et al. 2014).

Amastridium veliferum se distribuye desde Nuevo León (México) hasta el corregimiento de Guaduas, municipio del Carmen de Atrato, departamento del Chocó en Colombia, 1610-1690 msnm (Wilson y Meyer 1969, Savage 2002, Rueda-Almonacid y Rueda-Martínez 2004). A. veliferum es una especie inusual o poco avistada, debido a sus hábitos semifosoriales y de ambientes selváticos como bosques primarios, secundarios y en ocasiones se observan en pastizales; sin embargo, la historia natural de esta especie es desconocida (Wilson y Meyer 1969, Savage 2002, Rueda-Almonacid y Rueda-Martínez 2004). Algunos reportes de poblaciones de Tamaulipas (México) mencionan el consumo de pequeñas ranas del género Eleutherodactylus y una reproducción ovípara.

En el año 2010, se realizó una expedición en busca de anfibios y reptiles en el Darién Chocoano, por medio del Instituto de Investigaciones Ambientales del Pacífico (IIAP) y la Universidad Tecnológica del Chocó «Diego Luis Córdoba» (UTCH), donde se registró un macho adulto de $A$. veliferum, proveniente del Cerro Tacarcuna en el municipio de

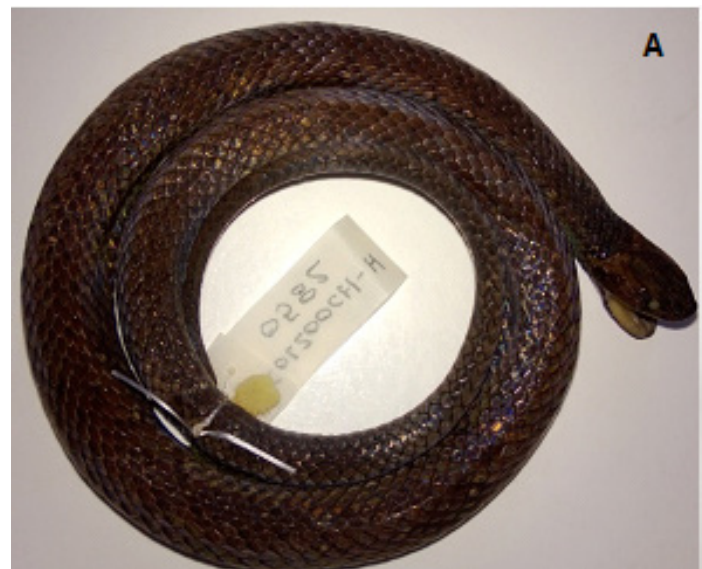

Unguía, departamento del Chocó, Colombia, a 1000 msnm ( $8^{\circ} 1$ '30'N, $\left.77^{\circ} 09^{\prime} 25^{\prime \prime} \mathrm{W}\right)$. El espécimen fue capturado en la mañana (9:20 am) del día 15 de septiembre de 2010 por medio del método de búsqueda por encuentros visuales (VES por sus siglas en inglés) (Crump y Scott 1994). Este macho se encontraba en reposo, sobre la hojarasca, en un bosque primario o maduro de mucha edad, además, se observaron ecosistemas con una gran variación de orquídeas, helechos, hongos, musgos y líquenes.

El espécimen se encuentra depositado en la Colección Científica de Referencia Zoológica del Chocó-Herpetología, UTCH, identificado con el número de colección UTCH: COLZOOCH-H 00582 (Figura 1).

Este ejemplar se asigna a $A$. veliferum por las siguientes características morfológicas, las cuales concuerdan con las características reportadas por Wilson y Meyer (1969), Smith (1971), Savage (2002) y Rueda-Almonacid y Rueda-Martínez (2004): presenta una cabeza alargada claramente diferenciable del cuerpo, con una longitud total de $404 \mathrm{~mm}$ y longitud cuerpo de $295 \mathrm{~mm}$, la cola mide $114 \mathrm{~mm}, 7 \mathrm{~mm}$ de ancho cefálico, $13 \mathrm{~mm}$ de longitud cefálica, $3 \mathrm{~mm}$ de distancia narina-ojo y $3 \mathrm{~mm}$ de diámetro del ojo. Presenta una escama rostral normal, dos escamas internasales, dos escamas prefrontales, dos escamas supraoculares, una frontal, dos escamas parietales, escama nasal dividida, loreal ausente, una escama preocular, dos postoculares, sin suboculares, uno a dos escamas temporales, ocho escamas supralabiales, las supralabiales 3 y 4 en contacto con la órbita del ojo,

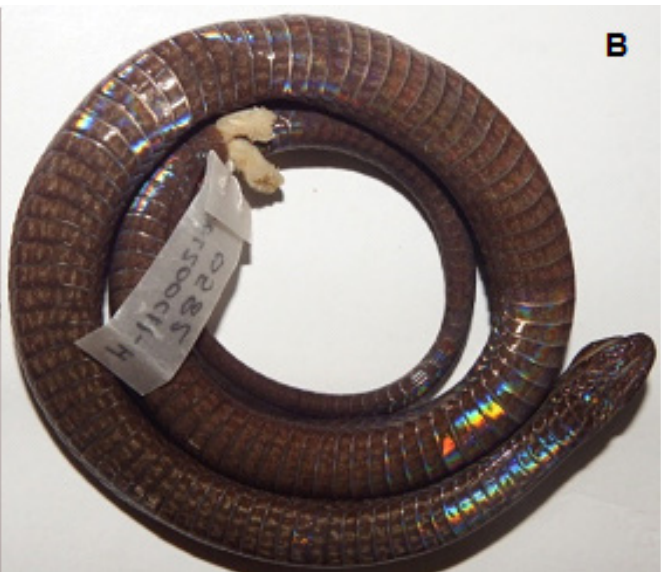

Figura 1. Vista dorsal (A) y vista ventral (B) del macho de Amastridium veliferum registrado en el Cerro Tacarcuna, municipio de Unguía, departamento del Chocó, Colombia y depositado en Colección Científica de Referencia Zoológica del Chocó-Herpetología, Universidad Tecnológica del Chocó «Diego Luis Córdoba» (UTCH: COLZOOCH-H 00582). 


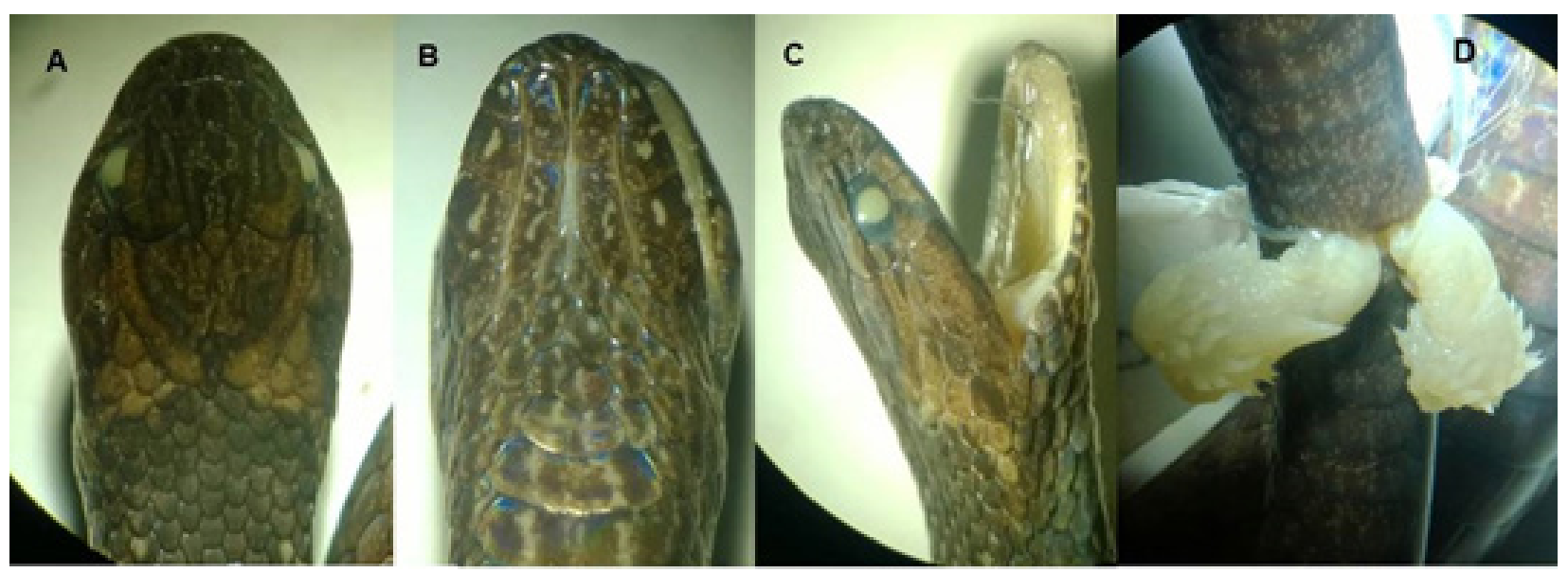

Figura 2. Esquema de los patrones de coloración de la cabeza (A) vista dorsal, (B) vista ventral, (C) vista lateral, y (D) hemipene de Amastridium veliferum colectado en el municipio de Unguía, Chocó, Colombia.

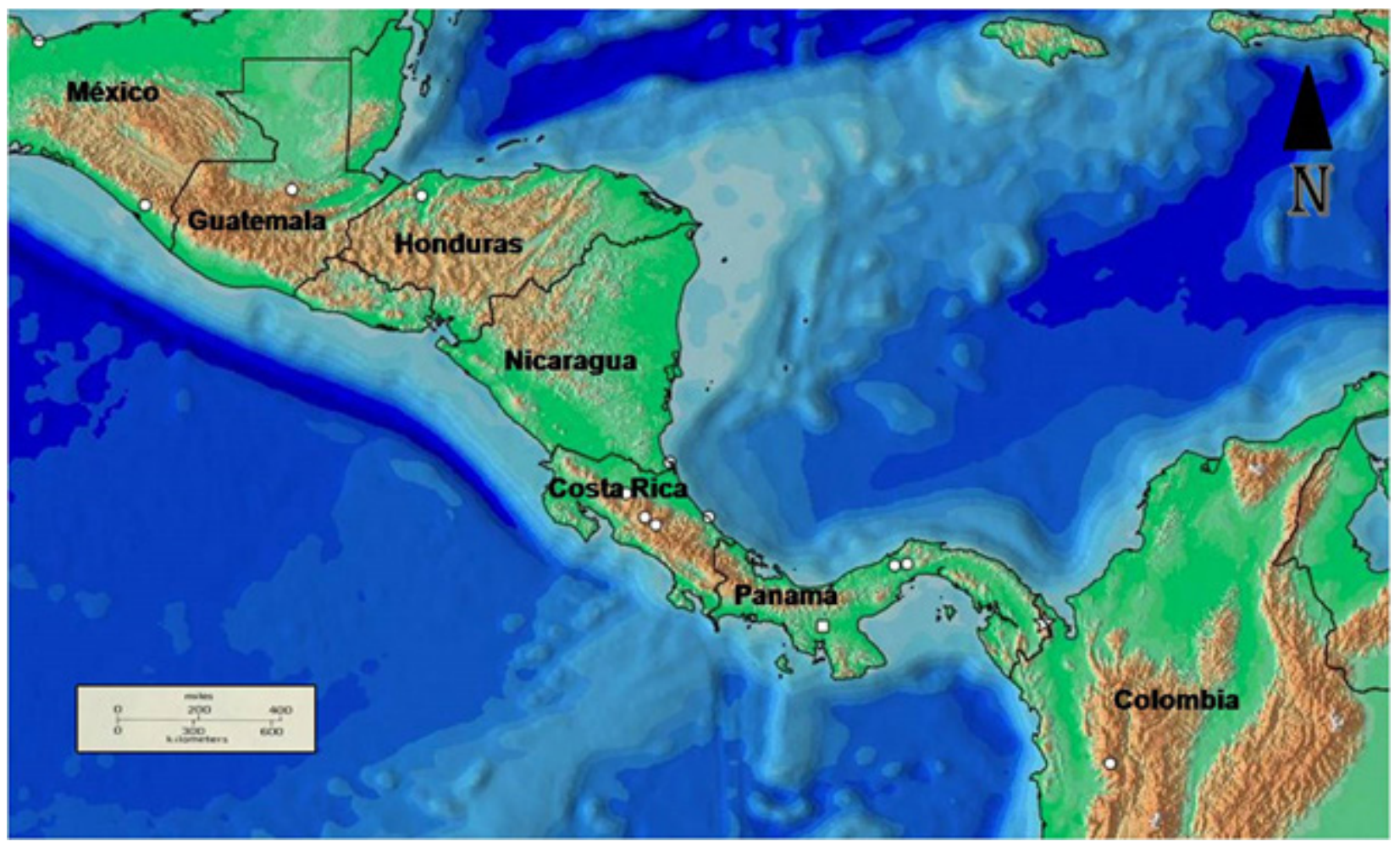

Figura 3. Distribución geográfica de Amastridium veliferum. Círculos: corresponde a los reportes de la literatura (Wilson y Meyer 1969, Rueda-Almonacid y Rueda-Martínez 2004). Cuadro: localidad tipo. Estrella corresponde al nuevo registro hecho en el presente trabajo. Mapa ubicado en la parte superior derecha pertenece a Colombia y al área de distribución de la especie en Colombia.

nueve escamas infralabiales, cuarta escama infralabial en contacto con el primer par de geniales, segunda escama infralabial en contacto con el segundo par de geniales y la quinta escama infralabial es la más grande (Figura 2A-2C), además, el dorso cefálico presenta el complemento de escudos característico de la familia. Las escamas dorsales son lisas y dispuestas en 17 filas a lo largo del tronco; 124 escamas ventrales, 53 escamas subcaudales divididas, placa anal dividida y supracloacales quilladas; fosetas apicales sólo en la región nucal. Los hemipenes evertidos son simples, claviforme no capitádo, con tres grandes espinas en 
la base, levemente bifurcado en el ápice, su tercio distal caliculado y los dos tercios basales espinosos, área por debajo de la triada grande, lisa y desnuda. Espinas de la superficie surcada algo más pequeña que las del lado asurcado. Cálices del lado surcado grande, papilados los pequeños y espinulados (Rueda-Almonacid y Rueda-Martínez 2004; Figura 2D).

El ejemplar en preservado (etanol 70\%) muestra una coloración dorsal gris marrón oscuro, con filas de pequeñas manchas blancas cada cuatro o cinco escalas en quinta fila; la región más dorsal de la cabeza tiene una tonalidad rojiza-café, presenta dos líneas longitudinales más visibles en la cabeza de color oscuro, partiendo de la escama rostral hacia atrás; pupila redondeada; vientre pardo grisáceo, más pálido hacia la región anterior.

Este espécimen macho representa el segundo registro de $A$. veliferum para Colombia y para el departamento del Chocó, porque en el año 1987 otro especímen de esta especie fue capturado en el corregimiento de Guaduas, municipio del Carmen de Atrato, entre los 1610 y $1690 \mathrm{msnm}\left(05^{\circ} 45^{\prime} 05^{\prime}\right.$ " $\mathrm{N}, 76^{\circ} 08^{\prime} 03$ " W). Este primer registro fue realizado por Rueda-Almonacid y Rueda-Martínez en el 2004, quienes extendieron la distribución del género hacia el sur en más de $500 \mathrm{~km}$ e incrementaron los límites de su distribución altitudinal (Figura 3).

Esta especie actualmente no se encuentra en el listado de la Convención sobre el Comercio Internacional de Especies Amenazadas de Fauna y Flora Silvestre (CITES 2014) y está clasificada en la categoría preocupación menor (LC por su sigla en inglés) de la Unión Internacional para la Conservación de la Naturaleza (IUCN 2014 por sus siglas en inglés).

El Cerro de Tacarcuna posee una vegetación bastante diferenciada de las zonas bajas que lo circundan las cuales han sido transformadas a causa de la expansión de la frontera agrícola y la ganadería; sin embargo, las partes altas del cerro están cubiertas con un bosque espeso donde predominan especies listadas en los libros rojos de Colombia como es el caso del roble (Quercus humboltdii) y muchas de las especies de angiospermas que allí conviven se encuentran restringidas a este importante ecosistema (Gradstein y Salazar 1992). El nuevo registro hecho aquí, revela que existen grandes probabilidades de encontrar otros y hasta novedades biológicas en el Cerro Tacarcuna; no obstante, los problemas de orden público a los que han sido objetos gran parte del territorio colombiano no han permitido la exploración adecuada de estas zonas, restringiendo el conocimiento de la biodiversidad.

\section{Agradecimientos}

Los autores agradecen a Paulo Passos (Departamento de Vertebrados, Museu Nacional, Univer-sidade Federal do Rio de Janeiro) a Guido Fabián Medina Rangel (Universidad Nacional de Colombia), Fernando Castro Herrera (Universidad del Valle), Teddy Angarita Sierra (Fundación YOLUKA) y a Marvin Anganoy-Criollo, por sus aportes en la taxonomía y al documento, al Instituto de Investigaciones Ambientales del Pacífico (IIAP), a Universidad Tecnológica del Chocó y por último al Grupo de Investigación en Herpetología de la Universidad Tecnológica del Chocó «Diego Luis Córdoba» por su apoyo moral.

\section{Literatura citada}

Acevedo ML, Wilson D, Cano EB, Vásquez-Almazán C. 2010. Diversity and conservation status of the Guatemalan herpetofauna. In: Conservation of Mesoamerican amphibians and reptiles. Wilson LD, Townsend JH, Johnson JD (eds.). Eagle Mountain: Eagle Mountain Publishing. pp. 406-35.

Cope ED. 1861. Descriptions of reptiles from tropical America and Asia. Proc Acad Nat Sci Philadelphia. 1860: 368-74.

Convención sobre Comercio Internacional de Especies Silvestres de Fauna y Flora (CITES). 2014. Lista de Especies CITES. www.cites.org/esp/app/2014/S-Appendices-2014-06-12. pdf

Crump ML, Scott NJ. 1994. Visual Encounter Surveys. In: Measuring and monitoring biological diversity. Standard methods for amphibians. Heyer W, Donnelley MA, McDiarmid RA, Hayec LC, Foster MC (eds.). Washington DC: Smithsonian Institution Press.

Gradstein SR, Salazar AN. 1992. Bryophyte diversity alongan altitudinal gradient Darien National Park. Trop Bryol. 5: 61-71.

IUCN. 2014. The IUCN Red List of Threatened Species. Version 2014.3. URL: http://www.iucnredlist.org (downloaded on 17 November 2014).

Johnson JD, Mata-Silva V, Ramírez-Bautista A. 2010. Geographic distribution and conservation of the herpetofauna of southeastern Mexico. In: Conservation of Mesoamerican amphibians and reptiles. Wilson LD, Townsend JH, Johnson JD (eds.). Eagle Mountain: Eagle Mountain Publishing. pp. 322-69.

Köhler G. 2008. Reptiles of Central America. $2^{\text {nd }}$ ed. Offenbach: Herpeton. 400 pp.

Lara-Tufiño D, Hernández-Austria R, Wilson LD, Berriozabal-Is- 
las C, Ramírez-Bautista A. 2014. New state record for the snake Amastridium sapperi (Squamata: Dipsadidae) from Hidalgo, Mexico. Rev Mex Biodivers. 85: 654-7.

McCranie JR. 2011. The snakes of Honduras: systematics, distribution, and conservation. Ithaca, Society for the Study of Amphibians and Reptiles, Contributions to Herpetology. Rev Mex Biodivers. 82: 1368-70.

Pyron RA, Burbrink FT, Wiens JJ. 2013. A phylogeny and revised classification of Squamata, including 4161 species of lizards and snakes. BioMed Central Evolut Biol. 13: 93.

Rueda-Almonacid JV, Rueda-Martínez JN. 2004. Primer registro de una serpiente del género Amastridium Cope (Serpentes: Colubridae), para Sudamérica. Rev Acad Colomb Cienc. 28 (107): 291-3

Savage JM. 2002. The amphibians and reptiles of Costa Rica: A herpetofauna between two continents, between two seas. Chicago: University of Chicago Press. 934 pp. Review in Copeia 2003 (1): 205.

Smith-Hobart M. 1971. The snake genus Amastridium in Oaxaca, Mexico. Great Basin Natur. 31: 254-5.

Stafford PJ, Meyer JR. 2000. A guide to the reptiles of Belize. London: Academic Press. 356 pp.

Staffor PJ, Walker P, Edgar P, Penn MG. 2010. Distribution and conservation of the herpetofauna of Belize. In: Conservation of Mesoamerican amphibians and reptiles. Wilson LD, Townsend JH, Johnson JD (eds.). Eagle Mountain: Eagle Mountain Publishing, LC. pp. 370-405.

Towsend JH, Wilson LD. 2010. Conservation of the Honduran herpetofauna: issues and imperatives. In: Conservation of Mesoamerican amphibians and reptiles. Wilson LD, Townsend JH, Johnson JD (eds.). Eagle Mountain: Eagle Mountain Publishing. p. 460-87.

Werner F. 1903. Ueber Reptil Und Batrachier Aus Guatemala und China in der zoologischen Staats-Sammulng in Münchennebsteinem Anhangüberseltene Formen ausanderen Gegenden. Abh Bayer Akad Wiss. 2: 343-84.

Wilson LD. 1988d. Amastridium, A. veliferum Cope. Catalogue of American Amphibians and Reptiles, pp. 449.

Wilson LD, Johnson JD. 2010. Distributional patterns of the herpetofauna of Mesoamerica, a biodiversity hotspot. In: Conservation of Mesoamerican amphibians and reptiles. Wilson LD, Townsend JH, Johnson JD (eds.). Eagle Mountain: Eagle Mountain Publishing. p. 30-235.

Wilson LD, Robinson DC. 1971. Additional specimens of the colubrid snake Amastridium veliferum (Colubridae) from Costa Rica, with comments on a pseudohermaphrodite. Bull So Calif Acad Sci. 70: 53-4.

Wilson LD, Meyer JR. 1969. A review of the colubrid snake genus Amastridium. Bull So Calif Acad Sci. 68 (3): 145-60.

Wilson LD, Mata-Silva V, Johnson JD. 2013. A conservation reassessment of the reptiles of Mexico based on the EVS measure. Amph Rept Conser. 7: 1-47.

Wilson LD, Meyer JR. 1985. The snakes of Honduras. $2^{\text {nd }}$ ed. Milwaukee Publ. Biol Geol N 6, 150 pp.

Uetz P, Hallermann J. 2014. The JCVI/TIGR Reptile Database. http://reptile-database.reptarium.cz/ 